\title{
Treatment Outcome of Tuberculosis and Associated Factors Among MTB/HIV Co-infected Patients in Sichuan, China: A Cross-Sectional Study (2016- 2020)
}

\section{Ni Yang}

Sichuan Center for Disease Control and Prevention

Chuang Chen ( $\square$ chuangchen_123@163.com )

Sichuan Center for Disease Control and Prevention

Jing Li

Sichuan Center for Disease Control and Prevention

\section{Yin Zhong}

Sichuan Center for Disease Control and Prevention

Jinge He

Sichuan Center for Disease Control and Prevention

\section{Lan Xia}

Sichuan Center for Disease Control and Prevention

\section{Linglin Zhang}

Sichuan Center for Disease Control and Prevention

\section{Wenfeng Gao}

Sichuan Center for Disease Control and Prevention

\section{Shu Zhang}

Sichuan Center for Disease Control and Prevention

\section{Research Article}

Keywords: Surveillance, MTB/HIV co-infected, Treatment outcome, China

Posted Date: January 24th, 2022

DOI: https://doi.org/10.21203/rs.3.rs-1150774/v1

License: (c) (1) This work is licensed under a Creative Commons Attribution 4.0 International License. Read Full License 


\section{Abstract}

Background: Mycobacterium tuberculosis/Human immunodeficiency virus (MTB/HIV) co-infection has increasingly become a pressing global public health problem. TB patients co-infected with HIV have poorer treatment outcome as compared to non-HIV-infected TB patients.This study was conducted to assess the outcomes of TB treatment and their associated factors among MTB/HIV co-infected patients in Sichuan, Southwest of China.

Methods: A cross-sectional study was performed among all of the MTB/HIV co-infected patients who were diagnosed and registered in TB designated hospitals and health facilities in Sichuan from $1^{\text {st }}$ January 2016 to $31^{\text {st }}$ December 2020. The data was collected from patients' electronic medical records regarding patients' demographic, clinical as well as their social support information. We calculated proportions of factors and treatment outcomes by Pearson's chi-square test and Fisher's exact test. Binary logistic regression model was fitted to identify factors associated with unsuccessful TB treatment outcome. For the logistic regression performed using alpha $=0.05$, odds ratios and corresponding $95 \%$ confidence intervals were calculated for the various risk factors.

Results: We included a total of 3183 registered MTB/HIV co-infected patients. Of the total patients, $89.54 \%$ had successful outcomes, $10.46 \%$ had unfavourable outcomes. Of the total successful outcomes, $21.49 \%$ were cured, $68.05 \%$ had completed their treatment. Being above 60 years old, received TB treatment at the municipal medical institute [AOR=2.093, 95\%Cl:1.219-3.593], diagnosed with EPTB [AOR=2.067, 95\% Cl:1.503-2.842], Relapsed or returned treatment after default [AOR=1.593, 95\%Cl:1.0482.420 ], being sputum smear positive [AOR=1.636, 95\% Cl: $1.290-2.075]$ were the factors that resulted the patients in unsuccessful treatment outcome.

Conclusion: Particular attention is recommended for MTB/HIV co-infected patients who are over 60 years old, receiving medical aid in the city-level medical institute, EPTB, relapsed or returned after default, sputum smear positive. National TB programme should strengthen social support and collaborative MTB/HIV management activities that would trace the identified factors in TB and HIV/AIDS designated health facilities to improve successful treatment outcome of TB.

\section{Background}

Tuberculosis among PLH is still a public health concern causing increased morbidity and mortality [1]. There were an estimated 1.2 million (range, 1.1-1.3 million) TB deaths among HIV-negative people in 2019 (a reduction from 1.7 million in 2000), and an additional 208000 deaths (range, 177 000-242 000) among HIV-positive people (a reduction from 678000 in 2000). In part because of the increasing number of people receiving ART [2]. The incidence of AIDS-related TB has decreased with the introduction of antiviral treatment, but the mortality of MTB/HIV patients is still much higher than that of TB patients without HIV infection or HIV/AIDS patients without TB. According to domestic reports, $20 \%-25 \%$ of those infected with MTB/HIV co-infection die of tuberculosis, which is the first cause of death in AIDS patients. 
People living with HIV/AIDS suffer from a loss of immunity and are susceptible to tuberculosis infection, potentially causing fatal harm. TB also has a negative impact on the immune response to HIV, increasing the progression from HIV infection to acquired immunodeficiency syndrome (AIDS). In the individual host, the two pathogens, Mycobacterium tuberculosis and HIV, accelerating the deterioration of immunological functions and resulting in premature death if untreated [3]. Moreover, the treatment success rate of TB coinfected with HIV is much lower. The treatment of patients with tuberculosis alone can achieve ideal curative effect, but the treatment success rate of patients with MTB/HIV co-infection is low. A study conducted in Lusaka [4], found that sociodemographic factors including poverty, being a woman, having EPTB, old age, marital status, and HIV co-infection are associated with unfavourable treatment outcomes. Recent studies have shown that HIV/AIDS patients with TB in Asian countries are more immunosuppressed and have a higher mortality rate than those in sub-Saharan Africa.

In China, although the AIDS epidemic has not yet led to a significant increase in the national tuberculosis epidemic, it has become a potential threat, especially with the increase in the number of AIDS patients and the diversification of AIDS epidemic patterns. According to WHO estimates, the two "HIV infection status surveys of TB patients" conducted in China from 2006 to 2008 showed that the prevalence of MTB/HIV co-infection is closely related to the prevalence of AIDS. In order to prevent and control MTB/HIV co-infection, China provides tuberculosis screening services to all PLH and HIV screening services for TB patients in key HIV/AIDS epidemic counties (cities). Sichuan Province is one of the areas with severe HIV epidemic in China. According to the data released at the AIDS academic exchange conference in October 2019, the number of new HIV infections and AIDS patients reported in January to October in 2019 increased the most in Sichuan Province, with more than 10,000 cases. The epidemic situation of MTB/HIV co-infection in Sichuan is also quite serious.

In MTB/HIV co-infected patients, HIV affects the effectiveness and success of TB treatment in many ways [5]. For example, the co-infected patients are exposed to many regimens including ART, anti-TB therapy, and preventive therapy of HIV-related co-morbidities which in turn is associated with an increased incidence of adverse drug reaction, poor adherence often due to pill burden, and decreased drug effectiveness. Consequently, the patient may experience a high rate leading to TB recurrence and increased risk of death [6]. These may impact or be associated with not only patients' clinical outcomes, bur also their beliefs about the HIV treatment, self-efficacy to the HIV treatment adherence, and their health status [7]. Hence, well-coordinated therapeutic management is needed to ensure optimum treatment outcomes in terms of response and prevention of drug resistance. Many MTB/HIV co-infected patients are unaware of their HIV infection status sometimes and although the AIDS patients complicated with TB are known to be HIV-positive, they have not finished their antiviral treatment yet, so they cannot take anti-tuberculosis drugs.

To reduce the burden of MTB/HIV among people living with HIV, it is recommended to scale up the three I's, which are intensified TB case-finding followed by high-quality TB treatment, IPT, and infection control for TB in all congregate settings and health facilities providing HIV care [8]. Currently, the treatment principles and treatment regimens for AIDS patients complicated with TB are the same as those for non- 
HIV-infected TB patients [9]. In addition, timely and effective anti-tuberculosis treatment is still the core measure to save the lives of patients. Identifying the risk factors that affect patients' treatment outcomes, carrying out bidirectional screening for TB and HIV, and starting timely and effective treatment can reduce the risk of relapsed or returned treatment after default and drug-resistant of TB, and further reduce mortality.

Most prior studies of treatment outcomes did very limited research on the demographic characteristics, clinical profiles in HIV co-infected TB patients in the area of Sichuan. Therefore, we determined the proportion of factors associated with unfavourable TB treatment outcomes in all designated TB medical institutions within Sichuan Province for the period 1st January 2016 to 31st December 2020.

\section{Material And Methods}

Design, setting, and population

Design and setting

A cross-sectional study design utilizing the Chinese National TB Surveillance System (NTSS) database was conducted. The system reports, analyse and utilize basic information, treatment information (such as treatment plan, treatment outcome, drug management, etc.) of patients diagnosed with TB, drug resistant TB, TB pleurisy, etc.

Population

The study population was comprised of all TB cases with HIV infection reported and registered in all designated TB medical institutions in Sichuan over a period of five years (2016-2020). Our study excluded those patients who initially were thought to have TB but were subsequently found to have a different diagnosis. In addition, medical records of who refused to accept TB treatment, not evaluated as well as patients who were transferred out were excluded since their TB treatment outcomes were unknown.

TB cases were defined as patients with either bacteriologically confirmed (smear and/or culture and/or histopathology and/or molecular biology-positive) or clinical diagnosed PTB, as well as EPTB. A patient was considered HIV infected when a screening test by ELISA tested positive or HIV strains isolated from blood or both results of screening test were positive at different times by a combination of clinical data. All TB patients under treatment follow up were tested for HIV. As soon as HIV is identified in a TB patient, the ART clinic started. For MTB/HIV co-infected patients, hospitals or health facilities serves DOTS both adult and pediatric age groups with free drugs like 2 months intensive phase taking Rifampicin, Isoniazid, Pyrazinamide and Ethambutol (2HRZE) and 4 months continuation phase taking Rifampicin and Isoniazid (4RH) [10].

Variable measurement 
We abstracted sociodemographic and clinical variables from the TB treatment registers that were related to the study objectives. The former included age, gender, ethnicity (Han, the other minority), occupation (unemployed, worker, farmer and other), level of medical institution (prefecture/city-level, county/districtlevel); The latter, The result of initial sputum smear test (positive, negative/not performed), type of entry (new cases, relapse or return after default), HIV antibody test result (known positive, new confirmed positive), TB form (PTB, EPTB, not performed), on ART (no, yes). In addition to standard outcome definitions, we classified the final TB treatment outcome as successful outcome (cured or treatment completed) vs unsuccessful outcome (died, failure, lost to follow up or not evaluated).

Data analysis and statistics

We entered abstracted data into Excel 2010 and exported to SPSS Version 17.0 for analysis. The continuous variable age was categorised as 4 different age groups. Pearson's chi-square tests or Fisher's exact tests were performed to compare groups in relation to the different level of medical institute. Frequency and percentage of categorical variables were reported. Measures of association for each category of the variables were estimated through Univariate and multivariable logistic regression models, with reference to the lowest expected risk category. The outcome variable was whether the individual had unsuccessful outcome or not after the treatment. For the logistic regressions performed using alpha $=$ 0.05 . Various risk factors were calculated using odds ratios at $p$-value $<0.05$ and $95 \%$ confidence intervals. Assumption of goodness of the model was checked by Hosmer-lemeshow test.

\section{Results}

There were 230,794 cases of TB reported and registered in Sichuan between 2016 and 2020, of which 3,672 cases were HIV co-infected. 489 Cases were excluded as they either missed information on TB treatment outcome or were found to have different diagnosis. Therefore, a total of 3,183 patients were included in the final analysis.

Table 1 shows the distribution of socio-demographic and clinical characteristics of MTB/HIV co-infected patients by level of medical institute in Sichuan from 2016 to 2020. The majority of the patients were male $2600(81.7 \%)$, of Han ethnicity $1872(58.8 \%)$, farmer $2595(81.5 \%)$, and the mean age was 43.82 (SD:14.282) year. The highest percentage of participants (42.3\%) were between 20 to 40 years of age.

$2169(68.1 \%)$ of the study participants were pulmonary TB. New cases constituted $94 \%$, while 2206 $(69.3 \%)$ of the participants were negative for the sputum smear test. Patients were known as positive for HIV antibody test constituted 76.6\%. 1906 (59.9\%) patients were not on ART. 3096 (97.3\%) of those had enrolled in county-level medical institute. The overall successful treatment rate for the two kinds of medical institute was $89.5 \%$.

The Univariate logistic regression model (Table 2) revealed that unsuccessful treatment outcome was more likely to occur in individuals aged over 60 years old (unadjusted odds ratio (UOR) $=2.669,95 \%$ Cl:1.045-6.816, $p=0.040)$, of The minority ethnicity ( $\mathrm{OR}=1.545,95 \% \mathrm{Cl}: 1.213-1.967, \mathrm{p}=0.000)$, and 
received care at the city-level medical institute $(\mathrm{OR}=2.476,95 \% \mathrm{Cl}$ : $1.469-4.172, \mathrm{P}=0.001)$. In addition, those who diagnosed with EPTB (OR=2.314, 95\% Cl: 1.696-3.157, $\mathrm{p}=0.000)$, relapsed or returned treatment after default ( $\mathrm{OR}=1.654,95 \% \mathrm{Cl}: 1.100-2.485, \mathrm{p}=0.016)$, and also with the initial sputum smear test positive ( $\mathrm{OR}=1.871,95 \% \mathrm{Cl}: 1.485-2.357, \mathrm{p}=0.000$ ) had increased risk of unsuccessful treatment outcome. Male MTB/HIV patients were less likely (OR=0.709, 95\% Cl: $0.514-0.979, p=0.037$ ) to develop unsuccessful treatment outcome compared to female MTB/HIV patients.

The multivariable logistic regression model (Table 3) showed that the odds of unsuccessful outcome were significantly low among MTB/HIV patients aged 21-40 years old (AOR=0.602, 95\% Cl:0.438-0.827, $\mathrm{p}=0.002)$ and $41-60$ years old $(\mathrm{AOR}=0.722,95 \% \mathrm{Cl}: 0.530-0.985, \mathrm{p}=0.040)$ compared to those aged above 60 years old. Meanwhile the odds were higher for MTB/HIV patients who sought medical aid at the citylevel medical institute (AOR=2.136, 95\% Cl: 1.250-3.651, $p=0.006)$ than county-level medical institute. Patients diagnosed with EPTB were more likely ( $A O R=2.115,95 \% \mathrm{Cl}: 1.541-2.903, p=0.000)$ to develop unsuccessful outcome compared to simple pulmonary TB patients. Relapsed or returned treatment after default patients had increased odds of unsuccessful outcome ( $A O R=1.588,95 \% \mathrm{Cl}: 1.047-2.407, p=0.029)$ compared to new cases. In addition, those whose initial sputum smear was positive (AOR=1.669, 95\% $\mathrm{Cl}: 1.317-2.114, \mathrm{p}=0.000$ ) had increased risk of unsuccessful outcome compared to those negative sputum smear examination. 
Table 1

Distribution of socio-demographic and clinical characteristics of MTB/HIV cases by level of medical institute. Sichuan, China, 2016-2020.

\begin{tabular}{|c|c|c|c|c|}
\hline \multirow[t]{2}{*}{ Characteristics } & \multicolumn{3}{|c|}{ level of medical institute } & \multirow{2}{*}{$\begin{array}{l}\mathrm{p} \\
\text { value }\end{array}$} \\
\hline & $\begin{array}{l}\mathrm{N}=3183 \\
(\%)\end{array}$ & $\begin{array}{l}\text { county-level,N=3096 } \\
\text { (\%) }\end{array}$ & $\begin{array}{l}\text { city- } \\
\text { level,N=87(\%) }\end{array}$ & \\
\hline Gender & & & & $0.253^{a}$ \\
\hline Male & 2600(81.7) & 2533(81.8) & $67(77.0)$ & \\
\hline Famale & $583(18.3)$ & $563(18.2)$ & $20(23.0)$ & \\
\hline Age & & & & $0.003^{b}$ \\
\hline $0-20$ & $82(2.7)$ & $81(2.6)$ & $1(1.1)$ & \\
\hline $21-40$ & $1348(42.3)$ & 1324(42.8) & $24(27.6)$ & \\
\hline $41-60$ & 1252(39.3) & 1214(39.2) & $38(43.7)$ & \\
\hline$>60$ & $501(15.7)$ & $477(15.4)$ & $24(27.6)$ & \\
\hline Ethnicity & & & & $0.000^{a}$ \\
\hline Han & 1872(58.8) & 1790(57.8) & $82(94.3)$ & \\
\hline The other minority & $1311(41.2)$ & 1306(42.2) & $5(5.7)$ & \\
\hline Occupation & & & & $0.000^{\mathrm{b}}$ \\
\hline Unemployed & $333(10.5)$ & $319(10.3)$ & $14(16.1)$ & \\
\hline Worker & $158(5)$ & $144(4.6)$ & $14(16.1)$ & \\
\hline Farmer & 2595(81.5) & $2541(82.1)$ & $54(62.1)$ & \\
\hline The other & $97(3)$ & $92(3.0)$ & $5(5.7)$ & \\
\hline TB form & & & & $0.112^{b}$ \\
\hline PTB & $2169(68.1)$ & 2115(68.3) & $54(62.1)$ & \\
\hline ЕРТВ & $334(10.5)$ & $319(10.3)$ & 15(17.2) & \\
\hline Not performed & $680(21.4)$ & $662(21.4)$ & 18(20.7) & \\
\hline Type of entry & & & & $0.309^{a}$ \\
\hline
\end{tabular}

${ }^{a} p$-value associated with Fisher's exact test

${ }^{\text {b}}$ Test of homogeneity of proportions based on Pearson's chi-square test 


\begin{tabular}{|c|c|c|c|c|}
\hline \multirow[t]{2}{*}{ Characteristics } & \multicolumn{3}{|c|}{ level of medical institute } & \multirow{2}{*}{$p_{\text {value }}$} \\
\hline & $\begin{array}{l}N=3183 \\
(\%)\end{array}$ & $\begin{array}{l}\text { county-level,N=3096 } \\
\text { (\%) }\end{array}$ & $\begin{array}{l}\text { city- } \\
\text { level,N=87(\%) }\end{array}$ & \\
\hline New case & 2992(94.0) & 2908(93.9) & $84(96.6)$ & \\
\hline $\begin{array}{l}\text { Relapse or return after } \\
\text { default }\end{array}$ & 191(6.0) & $188(6.1)$ & $3(3.4)$ & \\
\hline Initial sputum smear & & & & $0.086^{a}$ \\
\hline Negative/not performed & 2206(69.3) & 2153(69.5) & $53(60.9)$ & \\
\hline Positive & $977(30.7)$ & $943(30.5)$ & $34(39.1)$ & \\
\hline HIV antibody test result & & & & $0.000^{\mathrm{a}}$ \\
\hline Known positive & 2438(76.6) & 2386(77.1) & $52(59.8)$ & \\
\hline New confirmed positive & $745(23.4)$ & $710(22.9)$ & $35(40.2)$ & \\
\hline ART & & & & $0.387^{\mathrm{a}}$ \\
\hline ART-Yes & 1277(40.1) & $1246(40.2)$ & $31(35.6)$ & \\
\hline ART-No & 1906(59.9) & 1850(59.8) & $56(64.4)$ & \\
\hline Outcome & & & & $0.000^{\mathrm{a}}$ \\
\hline successful & 2850(89.5) & 2782(89.9) & 68(78.2) & \\
\hline unsuccessful & $333(10.5)$ & $314(10.1)$ & $19(21.8)$ & \\
\hline \multicolumn{5}{|c|}{${ }^{a} \mathrm{p}$-value associated with Fisher's exact test } \\
\hline
\end{tabular}


Table 2

Association between demographic/ clinic characteristics and treatment outcome among MTB/HIV patients in Sichuan, 2016-2020.

\begin{tabular}{|c|c|c|c|c|}
\hline Characteristics & $\begin{array}{l}\text { Successful } \\
\text { Outcome, } \mathrm{N}=2850 \\
(\%)\end{array}$ & $\begin{array}{l}\text { Unsuccessful } \\
\text { Outcome, } N=333(\%)\end{array}$ & UOR (95\% Cl) & $\begin{array}{l}\mathrm{p} \\
\text { value }\end{array}$ \\
\hline \multicolumn{5}{|l|}{ Gender } \\
\hline Famale & $536(18.8)$ & $47(14.1)$ & Ref & \\
\hline Male & 2314(81.2) & 286(85.9) & $0.709(0.514,0.979)$ & 0.037 \\
\hline \multicolumn{5}{|l|}{ Age } \\
\hline $0-20$ & $77(2.7)$ & $5(1.5)$ & Ref & \\
\hline $21-40$ & $1229(43.1)$ & 119(35.7) & $1.491(0.592,3.756)$ & 0.397 \\
\hline $41-60$ & 1117(39.2) & 135(40.5) & $1.861(0.740,4.680)$ & 0.187 \\
\hline$>60$ & 427(15.0) & $74(22.2)$ & $2.669(1.045,6.816)$ & 0.040 \\
\hline \multicolumn{5}{|l|}{ Ethnicity } \\
\hline Han & 1646(57.8) & 226(67.9) & Ref & \\
\hline The other minority & $1204(42.2)$ & 107(32.1) & $1.545(1.213,1.967)$ & 0.000 \\
\hline \multicolumn{5}{|l|}{ Occupation } \\
\hline Unemployed & 299(10.5) & $34(10.2)$ & Ref & \\
\hline Worker & $146(5.1)$ & 12(3.6) & $0.723(0.364,1.437)$ & 0.355 \\
\hline Farmer & 2313(81.2) & 282(84.7) & $1.072(0.736,1.561)$ & 0.716 \\
\hline The other & $92(3.2)$ & $5(1.5)$ & $0.478(0.182,1.258)$ & 0.135 \\
\hline \multicolumn{5}{|l|}{ Medical institute } \\
\hline county-level & 2782(89.9) & $314(10.1)$ & Ref & \\
\hline city-level & 68(78.2) & 19(5.7) & $2.476(1.469,4.172)$ & 0.001 \\
\hline \multicolumn{5}{|l|}{ TB form } \\
\hline PTB & 1971(69.2) & 198(59.5) & Ref & \\
\hline EPTB & 271(9.5) & 63(18.9) & $2.314(1.696,3.157)$ & 0.000 \\
\hline Not performed & $608(21.3)$ & $72(21.6)$ & $1.179(0.887,1.567)$ & 0.257 \\
\hline \multicolumn{5}{|l|}{ Type of entry } \\
\hline New case & 2689(94.4) & $303(91.0)$ & Ref & \\
\hline
\end{tabular}




\begin{tabular}{|c|c|c|c|c|}
\hline Characteristics & $\begin{array}{l}\text { Successful } \\
\text { Outcome,N=2850 } \\
\text { (\%) }\end{array}$ & $\begin{array}{l}\text { Unsuccessful } \\
\text { Outcome,N=333 (\%) }\end{array}$ & UOR (95\% Cl) & $\begin{array}{l}p \\
\text { value }\end{array}$ \\
\hline $\begin{array}{l}\text { Relapse or return } \\
\text { treatment after } \\
\text { default }\end{array}$ & 161(5.6) & $30(9.0)$ & $1.654(1.100,2.485)$ & 0.016 \\
\hline \multicolumn{5}{|c|}{ Initial sputum smear } \\
\hline $\begin{array}{l}\text { Negative/not } \\
\text { performed }\end{array}$ & 2018(70.8) & $188(56.5)$ & Ref & \\
\hline Positive & $832(29.2)$ & $145(43.5)$ & $1.871(1.485,2.357)$ & 0.000 \\
\hline \multicolumn{5}{|l|}{$\begin{array}{l}\text { HIV antibody test } \\
\text { result }\end{array}$} \\
\hline Known positive & $2190(76.8)$ & $248(74.5)$ & Ref & \\
\hline $\begin{array}{l}\text { New confirmed } \\
\text { positive }\end{array}$ & $660(23.2)$ & $85(25.5)$ & $1.137(0.876,1.477)$ & 0.335 \\
\hline \multicolumn{5}{|l|}{ ART } \\
\hline ART-Yes & 1156(40.6) & $121(36.3)$ & Ref & \\
\hline ART-No & 1694(59.4) & $212(63.7)$ & $1.196(0.945,1.513)$ & 0.137 \\
\hline
\end{tabular}


Table 3

Socio-demographic and clinical factors associated with treatment outcome in MTB/HIV patients. Sichuan, 2016-2020.

\begin{tabular}{|c|c|c|}
\hline Characteristic & AOR (95\% Cl) & $\mathrm{p}$ value \\
\hline \multicolumn{3}{|l|}{ age } \\
\hline $0-20$ & $0.434(0.168-1.118)$ & 0.084 \\
\hline $21-40$ & $0.602(0.438-0.827)$ & 0.002 \\
\hline $41-60$ & $0.722(0.530-0.985)$ & 0.040 \\
\hline$>60$ & Ref & \\
\hline \multicolumn{3}{|l|}{ Medical institute } \\
\hline county-level & Ref & \\
\hline city-level & $2.136(1.250-3.651)$ & 0.006 \\
\hline \multicolumn{3}{|l|}{ TB form } \\
\hline PTB & Ref & \\
\hline EPTB & $2.115(1.541-2.903)$ & 0.000 \\
\hline Not performed & $1.078(0.807-1.439)$ & 0.613 \\
\hline \multicolumn{3}{|l|}{ Type of entry } \\
\hline New case & Ref & \\
\hline $\begin{array}{l}\text { Relapse or return treatment } \\
\text { after default }\end{array}$ & $1.588(1.047-2.407)$ & 0.029 \\
\hline \multicolumn{3}{|l|}{ Initial sputum smear } \\
\hline Negative/not performed & Ref & \\
\hline Positive & $1.669(1.317-2.114)$ & 0.000 \\
\hline
\end{tabular}

\section{Discussion}

Tuberculosis and HIV co-infection remains one of the most public health challenges in Southwest of China. In the study, we found an overall TB treatment success rate of $89.5 \%$ for 3183 TB patients living with HIV in five years (2016-2020), which is higher than the national treatment successful rate of $76.0 \%$ in 2018, and is nearly in agreement with studies conducted in Asian countries like in Vietnam 74.0\% [11]; in Indian 80.0\% [12]; in Thailand 74.3\% [13]. However, this rate affects the target of achieving the 90\% TB treatment success rate for TB patients set by Global Plan to stop TB 2011-2015 [14]. 
Based on their self-descriptions, the majority of the TB patients living with HIV were males which is in line with findings from other literature [15]. Majority, at $42.3 \%$ and $39.3 \%$ of MTB/HIV patients were in the age groups of 21-40 and 41-60 years respectively. This report is also in agreement with studies by Mekonnen D et al [16]. Most of the patients were of Han ethnicity and farmers. This study also revealed high proportions of MTB/HIV among new cases, diagnosed with PTB, sputum smear-negative TB, HIV antibody positive and received ART.

Univariate regression analysis showed that male, aged above 60 years old, of Han ethnicity, sought medical aid at the city-level of medical institute, EPTB, relapse or return treatment after default and positive initial sputum smear result were the risk factors for unsuccessful treatment outcome. However, the odds of gender and ethnicity were not statistically significantly different among MTB/HIV patients compared to the reference group in the multivariable regression model.

This study showed that patients aged exceed 60 years old had higher odds of developing poorer outcomes compared to other age groups. The effect of age on treatment outcome may be more sophisticated to explain. As a matter of fact, many studies have had a similar result that advanced age is associated with unsuccessful outcome in TB treatment [17]. Not surprisingly, advanced age is a risk factor for mortality among TB patients with or without HIV. In part this finding could be contributed to age-related decreases in immunity [18], making a person more susceptible to infection, as growing older may lead to poor outcomes [19]. In addition, this findings are based on secondary data from routine surveillance, so we could not control for the age-related confounding variables.

In this study, the medical institute where a MTB/HIV patient sought medical aid was found to be linked with unsuccessful outcome. The odds of having unsuccessful TB treatment outcome was 2.136 times higher among patients seeking medical aid at the city-level medical institutes than that at the county-level institutes. This is similar to earlier reports from a study conducted in Zambia [20] and a study done in northern Ethiopia [21]. MTB/HIV co-infection patients who visit county-level medical institutions mostly seek medical advice after they have high-risk behaviors that may be infected with HIV, so that they can know their HIV infection status timely and be diagnosed early. Then they can receive antiviral and antituberculosis treatment nearby. On the contrary, the patients who choose to seek treatment in municipal medical institutions might because their immune system has been severely damaged, and opportunistic infections or AIDS-related diseases are discovered when they seek medical treatment, which is late and severe, thus affecting the treatment effect of patients with dual infection. This is an area that needs to be studied in the future to see what risk factors exist in different locations that may put people at higher risk of unfavourable outcomes in the TB treatment.

The MTB/HIV patients on the relapsed or returned treatment after default category had a higher chance of unsuccessful treatment outcome than the new cases. This result was similar to that reported from Brazil [22]. Mycobacterial resistance, a direct consequence of having been exposed to lack of treatment adherence or lost to follow up that contributes a lot to these unfavourable treatment outcomes. Hence, it should be aware of that adherence to treatment is one of the most important topics in the public policy 
agenda in view of the difficulty to manage the double burden of disease [23]. Nevertheless, a study conducted in Nigeria did not find any association between new and relapse patients [24].

Regarding the site of TB infection in the NTSS, cases registered as EPTB were $111.5 \%$ more likely to develop unsuccessful outcome than PTB $(p<0.05)$. Higher levels of immunosuppression increase the likelihood of EPTB or simultaneous EPTB and PTB, all of that are hard to cure [25]. Another study showed that EPTB is more common in the HIV-associated TB population, it becomes increasingly prevalent with progressive immunodeficiency, and it contributes to unfavorable treatment outcomes [26].

Finally, our study found that the chance of unsuccessful outcome was higher among MTB/HIV coinfected patients with sputum smear positive compared with the smear negative. Similar findings were reported by other studies conducted in South India [27] and Eastern Ethiopia [28]. In contrast, a study conducted in Mizan Tepi [29] demonstrated MTB/HIV co-infected patients with Smear positive PTB had a higher chance of successful TB treatment outcome.

A big strength of the study is that the large sample size allowed us to identify independent risk factors for unsuccessful treatment outcomes. In addition, the study was carried out under routine conditions, meaning the data were representative of the real situation, thus, the findings are generalizable to most of the populous province in China that accounts for a large proportion of PTB patients with HIV co-infected in the country.

Apart from such important findings, this study is not without limitation. One limitation is that the study included only patients who had an initial smear result of either positive or negative. And as common for secondary data based studies, important variables like CD4 level, distance to the health facility, level of education, $\mathrm{BMI}$ and economic status that could improve the model predictions were incomplete from the records. Another one is that we should acknowledge that there was a large amount of missing data regarding TB treatment outcome. These missing data could have biased our risk estimates. Bias is always a concern with secondary data sources, but the database of the NTSS has already served as a reliable source for several other population-based studies in China.

\section{Conclusion}

In this study, the overall TB treatment success rate was less than the WHO target of $>90 \%$. Moreover, MTB/HIV co-infected patients aged over 60 years old, being a relapse or returned treatment after default case, being TB sputum smear positive, being infected with EPTB and receiving care from the city-level medical institute were significantly associated with unsuccessful TB treatment outcome in all designated TB hospitals in Sichuan province. Thus, we would like to put forward a few recommendations as follows: (1) Especially attention should be paid to PTB patients co-infected with HIV who were above 60 years old, smear positive, relapsed or returned treatment after default, EPTB and who sought medical care at citylevel hospitals, which could improve the unsuccessful treatment outcome. (2) There is a pressing need for coordination between TB and HIV control programs in Southwest of China, through collaborative MTB/HIV activities. 


\section{Abbreviations}

National TB Surveillance System $₫$ NTSS; ART:Antiretroviral Therapy; BMI: Body mass index;CD4:Cluster of Differentiation 4; Cl: Confidence interval; AOR: Adjusted Odds Ratio; DOT: Directly Observed Therapy; EPTB: Extra-pulmonary TB; PTB: Pulmonary TB; SD: Standard Deviation; TB: Tuberculosis; MTB/HIV: Mycobacterium Tuberculosis/ Human Immunodeficiency Virus; ELISA: enzyme-linked immunosorbent assay; WHO: World health organization; IPT: Isoniazid preventive therapy; PLH: People living with Human Immunodeficiency Virus

\section{Declarations}

\section{Availability of data and materials}

The dataset supporting the conclusions of this article is available in the Github repository, https://github.com/Sccdc/sccdctb/blob/main/tbhivdata.sav

\section{Acknowledgments}

The authors would like to thank the data collectors, hospital staff working at TB clinics and heads of hospitals for their willingness and unreserved contribution in this study.

\section{Founding}

No funding from any source was obtained for this study.

\section{Author information}

$\mathrm{Ni}$ Yang is first author

Affiliations

${ }^{1}$ Sichuan Center for Disease Control and Prevention, Chengdu 610041, Sichuan Province, China.

Ni Yang, Jing Li, Yin Zhong, Wenfeng Gao, Jinge He, Lan Xia, Chuang Chen, Linglin Zhang

Contributions

$\mathrm{Ni}$ Yang designed this study, performed the statistical analyses and data interpretation and wrote the main manuscript. Jing Li, Yin Zhong contributed to data acquisition. Wenfeng Gao and Shu Zhang contributed to interpretation of laboratory data. Chuang Chen contributed to manuscript revisions. Jinge $\mathrm{He}$, Lan Xia, Linglin Zhang supervised this study. All authors reviewed the manuscript.

Corresponding author

Correspondence to Chuang Chen. 


\section{Ethics declarations}

Ethics approval and consent to participate

Before reviewing medical records of the MTB/HIV co-infected patients, permission was obtained from the TB treatment unit heads of the hospital. Patient records/ information was anonymized and de-identified prior to analysis. All methods used in this study were carried out in accordance with relevant guidelines and regulations and approval for conducting this study was obtained from Ethics Committee of Sichuan Center for Disease Control and Prevention (SCCDCIRB 2021-026).

\section{Consent for publication}

Not applicable.

\section{Competing interests}

The authors declare that they have no competing interests.

\section{References}

1. Dravid Ameet, Natarajan Kartik, Medisetty Mahenderkumar et al. Incidence of tuberculosis among HIV infected individuals on long term antiretroviral therapy in private healthcare sector in Pune, Western India.[J]. BMC Infect Dis, 2019, 19: 714.

2. WHO. Global tuberculosis report 2020 Geneva: World Health Organization; 2020.

3. Bruchfeld J, Correia-Neves M, Källenius G.Tuberculosis and HIV Coinfection.[J].Cold Spring Harb Perspect Med, 2015,5: a017871.

4. Kapata N, Chanda KP, Michelo C. The social determinants of tuberculosis and their association with TB/HIV co-infection in Lusaka, Zambia. Medical Journal of Zambia. 2013; 40 (2).

5. WHO. Global tuberculosis report 2019 Geneva: World Health Organization; 2019.

6. Frontières MS, Partnership ST. OUT OF STEP 2017. TB policies in 29 countries. A survey of prevention, testing and treatment policies and practices July 2017.

7. Yujia Zhu, MM, Jizhou Wu,et al. Patient characteristics and perceived health status of individuals with HIV and tuberculosis coinfection in Guangxi, China.Medicine (2017) 96:14.

8. WHO. WHO policy on collaborative TB/HIV activities: Guidelines for national programs and other stakeholders. World Health Organization 2012.

9. Department of Disease Control, $\mathrm{MOH}$, Department of Medical Administration, $\mathrm{MOH}$,Chinese Center for Diease Control and Prevention. Guidlines for Implementing the National Tuberculosis Control Program in China,2008:86-87. 
10. Zou G, Wei X, Walley JD, et al. Factors influencing integration of TB services in general hospitals in two regions of china: a qualitative study. BMC Health Serv Res 2012;12:1.

11. Huyen TTT, Nhung NV, Shewade HD, Hoa NB, Harries AD. Collaborative activities and treatment outcomes in patients with HIV-associated tuberculosis in Viet Nam. Public Health Action. 2016;6(1):8-14.

12. Ambadekar NN, Zodpey SP, Soni RN, Lanjewar SP. Treatment outcome and its attributes in TB-HIV coinfected patients registered under revised national TB control program: a retrospective cohort analysis. Public Health. 2015;129:783e9.

13. Gatechompol S, Kawkitinarong K, Suwanpimolkul G, et al. Treatment outcomes and factors associated with mortality among individuals with both TB and HIV in the antiretroviral era in Thailand. J Virus Erad. 2019;5(4):225-230.

14. World Health Organization, Stop TB Initiative (World Health Organization)Treatment of Tuberculosis: Guidelines. 2010;World Health Organization.

15. Murphy ME, Wills GH, Murthy S, Louw C, Bateson ALC, Hunt RD et al. Gender differences in tuberculosis treatment outcomes: a post hoc analysis of the REMoxTB study. BMC Med. 2018; 16(1): 189.

16. Mekonnen D, Derbie A, Mekonnen H, Zenebe Y. Profile and treatment outcomes of patients with tuberculosis in Northeastern Ethiopia: a cross sectional study. Afr Health Sci. 2016;16(3):663-670.

17. Liew SM, Khoo EM, Ho BK, Lee YK, Mimi O, Fazlina MY et al. Tuberculosis in Malaysia: predictors of treatment outcomes in a national registry. Int J Tuberc Lung Dis. 2015; 19(7): 764-71.

18. Weinberger B, Herndler-Brandstetter D, Schwanninger A, Weiskopf D, Grubeck-Loebenstein B. Biology of immune responses to vaccines in elderly persons. Clin Infect Dis. 2008;46:1078-84.

19. Mok J, An D, Kim S, Lee M, Kim C, Son H. Treatment outcomes and factors affecting treatment outcomes of new patients with tuberculosis in Busan, South Korea: a retrospective study of a citywide registry, 2014-2015. BMC Infect Dis. 2018; 18(1): 655.

20. Francis Hamaimbo Nanzaluka1,2,\&, Sylvia Chibuye2, Clara Chola Kasapo, et al.Factors associated with unfavourable tuberculosis treatment outcomes in Lusaka, Zambia, 2015: a secondary analysis of routine surveillance data.Pan African Medical Journal. 2019;32:159.

21. Tesfahuneygn G, Medhin G, Legesse M. Adherence to Anti-tuberculosis treatment and treatment outcomes among tuberculosis patients in Alamata District, Northeast Ethiopia. BioMed Central. 2015; 8: 503.

22. Prado TNd, Rajan JV, Miranda AE, Dias EdS, Cosme LB, Possuelo L Ga, et al. Clinical and epidemiological characteristics associated with unfavorable tuberculosis treatment outcomes in TBHIV co-infected patients in Brazil: a hierarchical polytomous analysis. Braz J Infect Dis 2017;21(2):162-170.

23. Mabunda T E, Ramalivhana NJ, Dambisya YM. Mortality associated with tuberculosis/HIV coinfection among patients on TB treatment in the Limpopo province, South Africa. Afr Health Sci. $2014 ; 14(4): 849-854$. 
24. Ukwaja KN, Sarah N Oshi, Isaac Alobu, and Daniel C Oshi. Profile and determinants of unsuccessful tuberculosis outcome in rural Nigeria: Implications for tuberculosis control. World J Methodol. 2016; 6(1): 118-25.

25. Ronaidi NN, Mohd NS, Wan Mohammad Z, Sharina NR, NikRosmawati NH. Factors associated with unsuccessful treatment outcome of pulmonary tuberculosis in Kota Bharu, Kelantan. Malays $\mathrm{J}$ Public Health Med. 2011;11:6-15.

26. Arpagaus, A.; Franzeck, F.C.; Sikalengo, G.; Ndege, R.; Mnzava, D.; Rohacek, M.; Hella, J.; Reither, K.; Battegay, M.; Glass, T.R.; et al. Extrapulmonary tuberculosis in HIV-infected patients in rural Tanzania: The prospective Kilombero and Ulanga antiretroviral cohort. PLoS ONE 2020, 15, e0229875.

27. Vijay S, Kumar P, Chauhan LS, Rao SVN, Vaidyanathan P. Treatment outcome and mortality at one and half year follow-up of HIV infected TB patients under TB control Programme in a district of South India. PLoS One. July 2011;6(7):1-8.

28. Assefa Tola1, Kirubel Minsamo Mishore, Yohanes Ayele, Abraham Nigussie Mekuria and Nanati Legese.Treatment Outcome of Tuberculosis and Associated Factors among TB-HIV Colnfected Patients at Public Hospitals of Harar Town, Eastern Ethiopia. A five-year retrospective study. BMC Public Health (2019) 19:1658.

29. Kefale AT, Anagaw YK. The outcome of tuberculosis treatment and its predictors among HIV infected patients in Southwest Ethiopia. Int J Gen Med. 2017;10:161-70. 